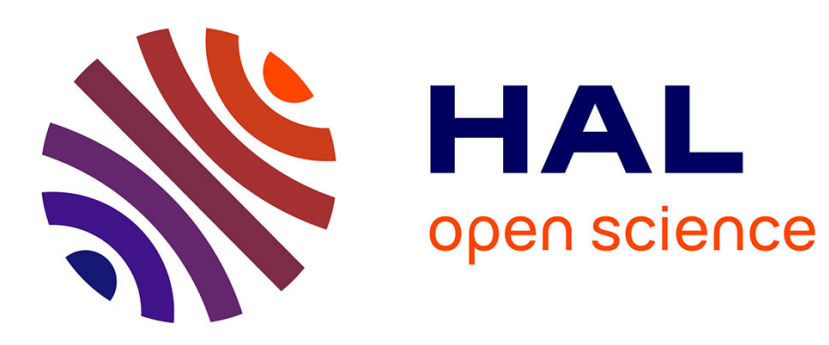

\title{
FPGA Acceleration of the Horn and Schunck Hierarchical algorithm
}

Ilias Bournias, Roselyne Chotin, Lionel Lacassagne

\section{To cite this version:}

Ilias Bournias, Roselyne Chotin, Lionel Lacassagne. FPGA Acceleration of the Horn and Schunck Hierarchical algorithm. International Symposium on Circuits and Systems (ISCAS), May 2021, Daegu, South Korea. 10.1109/ISCAS51556.2021.9401068 . hal-03330803

\section{HAL Id: hal-03330803 https://hal.science/hal-03330803}

Submitted on 1 Sep 2021

HAL is a multi-disciplinary open access archive for the deposit and dissemination of scientific research documents, whether they are published or not. The documents may come from teaching and research institutions in France or abroad, or from public or private research centers.
L'archive ouverte pluridisciplinaire HAL, est destinée au dépôt et à la diffusion de documents scientifiques de niveau recherche, publiés ou non, émanant des établissements d'enseignement et de recherche français ou étrangers, des laboratoires publics ou privés. 


\title{
FPGA Acceleration of the Horn and Schunck Hierarchical Algorithm
}

\author{
Ilias Bournias*, Roselyne Chotin* and Lionel Lacassagne* \\ *Sorbonne Université, CNRS, LIP6, F-75005 Paris, France \\ firstname.surname@lip6.fr
}

\begin{abstract}
This work proposes a highly tunable motion estimation architecture. We implement the Horn and Schunck algorithm with the hierarchical extension for larger motion estimations in FPGAs. Different architectures are explored dealing with interpolation, pipeline, parallelism and arithmetic format, in order to fit performance. We show in our exploration, how the different cores of our system should be used to increase the throughput. Our smallest design achieves a $30.8 \mathrm{Mpixel} / \mathrm{s}$ in a $\mathbf{1 0 2 4} \times \mathbf{1 0 2 4}$ resolution and the fastest $507 \mathrm{Mpixel} / \mathrm{s}$ which is one of the fastest ever achieved, as far as we know, for FPGAs.
\end{abstract}

\section{INTRODUCTION}

\section{A. State-of-the-Art}

Optical flow algorithms are used to estimate the velocity of each pixel between a pair of images. These algorithms are used in a variety of applications from object detection, motion compensation, to autonomous driving.

Most of the literature focuses on the accuracy [1], and only few target embedded systems and address the tradeoffs that one has to do for a realtime implementation, namely the number of iterations, the computing format (the number of bits of fixed or floating point number), and the parallelism.

There are a lot of optical flow algorithms according to their typically organized according to their computational speed, accuracy and specific application.

The estimation of the optical flow in real time is a challenging task because it requires a lot of computation efforts and in the same time the hardware to remain low. There were a lot of works in optimizing optical flow algorithms in CPU [2]-[4], GPU [5]-[7] and FPGAs. Especially for FPGAs, some works use the Lucas-Kanade $(L \& K)$ method with mono-scale and multi-scale implementations for [8] while [9] remains on the multi-scale Phase-based algorithm and [10] proposes a lower frame memory access to reduce external memory interactions. Finally the works [11]-[13] implement a Horn and Schunck optical flow mono-scale algorithm, the first in an iterative mode and the others also in partial and fully pipelined modes.

\section{B. Horn and Schunck algorithm}

The basic scheme of Horn and Shunck $(H \& S)$ [14] is an iterative algorithm (Fig. 1(a)) that estimates $(u, v)$ from the first spatio-temporel derivatives $I_{x}, I_{y}, I_{t}$ (of a pair of images) and from the previous average values $(\bar{u}, \bar{v})$, according to (1) and (2) where $\alpha$ is a smoothing parameter.

$$
\begin{aligned}
& u=\bar{u}-I_{x} \frac{I_{x} u+I_{y} v+T_{t}}{\alpha^{2}+I_{x}^{2}+I_{y}^{2}} \\
& v=\bar{v}-I_{y} \frac{I_{x} u+I_{y} v+T_{t}}{\alpha^{2}+I_{x}^{2}+I_{y}^{2}}
\end{aligned}
$$

As the derivatives are estimated with a $2 \times 2 \times 2$ kernel, the computed velocities should be smaller than 1 pixel / frame. That is the reason why, multi-scale (aka hierarchical) scheme should be considered (Fig. 1(b)).

From the computed velocities $(u, v)_{\text {final }}^{\lambda+1}$ of level $\lambda+1$, a new velocity field is initialized : $(u, v)_{\text {init }}^{\lambda}$ by up-scaling the previous one with a factor 2 , and multiplying it also by a factor 2: $(u, v)_{\text {init }}^{\lambda}=2 \times \operatorname{Upscale}(u, v)_{\text {final }}^{\lambda+1}$. These velocities are used to compensate (warp) the motion between the two images $\left(I_{2 \text { rec }}\right)$, thanks to a bi-linear or bi-cubic interpolation. Then $H \& S$ kernel iterates to provide the residual velocities $(\delta u, \delta v)$. After the iterations these residuals are accumulated to the initial estimation: $(\mathrm{u}, \mathrm{v})_{\text {final }}^{\lambda}=(u, v)_{\text {init }}^{\lambda}+(u, v)^{\lambda}$ to provide the final velocity estimations at this level. Then same computations are done for the next level: $(u, v)_{\text {init }}^{\lambda-1}=2 \times \operatorname{Upscale}(u, v)_{\text {final }}^{\lambda}$ and so on until level $\lambda=0$.

In this paper, we first present an architecture that implements the multi-scale $H \& S$ algorithm in FPGA. As this architecture is highly tunable, section III will describe a design space exploration methodology to reach the performance whose results are discussed in section IV. Then, we conclude and explore future works.

\section{Contributions}

Instead of setting a constant number of iteration for every level, the convergence is better if one puts a high number of iterations at coarse level (small images) and low number of iterations at fine level (large images). Thus a very standard configuration with a 3 -level pyramid with a $\times 2$-factor $(20,10,5$ iterations) has been selected for our implementations which achieves a motion estimation in the range of $(V, U)<|7|$, but a $\times 4$-factor $(80,20,5$ iterations $)$ can be also considered. The image size is set at $1024 \times 1024$ pixels but can be adapted in other sizes too.

Our contribution is to propose a multi-scale implementation of $H \& S$ where the parameters to explore are the parallelism $(1,5,10$ or 20 computing kernels), the floating point format (16bit or 32-bit IEEE floating points) and the warp interpolation (bi-linear or bi-cubic). So we do not try to address accuracy, 


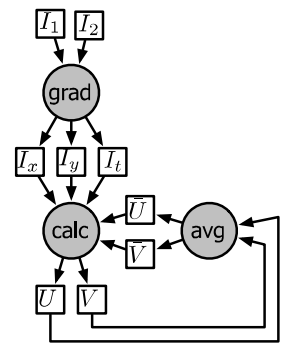

(a) H\&S mono-scale itera- (b) $\mathrm{H} \& \mathrm{~S}$ multi-scale iterative tive scheme

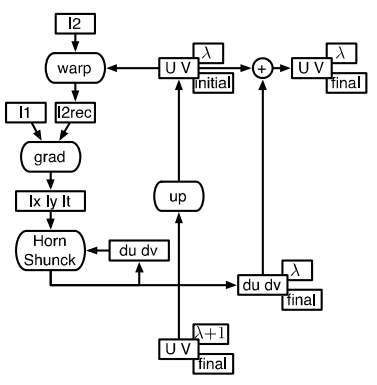

scheme at level $\lambda$
Fig. 1. Description of the Algorithm

as the convergence depends on the observed scene, we only evaluate the impact of the precision (16 or 32 bits) on the size of the design.

\section{Proposed Architecture}

In this section we will describe the different cores used in our design.

\section{A. Horn and Schunck core}

In this paper, we propose four different designs (Fig.2) depending on the number of $H \& S$ cores used and the number of iterations for each pyramid level. The first three implementations are the standard iterative $(I)$, the partial pipeline $(P)$ and fully pipeline $(F)$ used by [12], [13]. The last one is a fully pipeline parallel implementation with $\pi$ parallel cores $\left(F_{\pi}\right)$. In the last case the $\pi$ parallel core is mandatory to access to $3 \times(\pi+2)$ neighboured previous average values $(\bar{u}, \bar{v})$, instead of the $3 \times 3$ required for the first 3 designs. With this design $\pi$ pixels per clock cycle can be processed.

\section{B. Warping core}

For the warping core two cases have been explored, the bi-linear and bi-cubic interpolation. For the bi-linear (resp. bicubic) interpolation, a neighbourhood of $2 \times 2$ (resp. $4 \times 4$ ) pixels in the input image is required. The goal is to interpolate one pixel per clock cycle, so we have to ensure that in every clock cycle all the required neighboured pixels are available in a similar way as in [15]. To do that the worst case scenario has to be examined. It occurs when the optical flow vectors summed from levels 2 and 1 have to be interpolated with the input image in level $0((V, U)<|6|)$. This means that for bi-linear (resp. bi-cubic) interpolation, 14 (resp. 16) lines have to be stored in 14 (resp. 16) FIFOs. In every clock cycle a new pixel is read from the external memory and all the remaining pixels inside the FIFOs are moved one position to the right so that all the required pixels for the interpolation are available without latency. In order to choose the right neighbouring pixels in the two cases, the integer parts of the velocity vectors are needed and the interpolation is done with the fraction part of the velocities. In order to process $\pi$ pixels per clock cycle, we have to parallelize the computation. In that case, $\pi$ pixels per clock cycle are read from the memory.

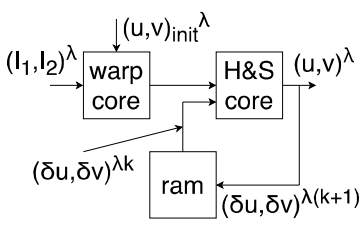

(a) Iterative $(I)$ Mode

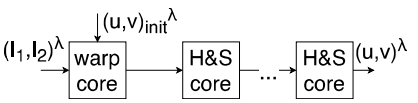

(c) Fully Pipeline $(F)$ Mode

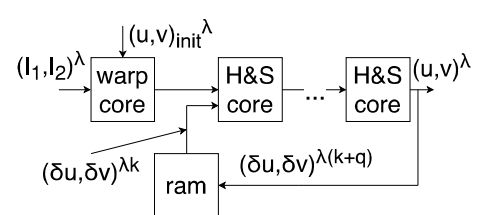

(b) Partial Pipeline $(P)$ Mode

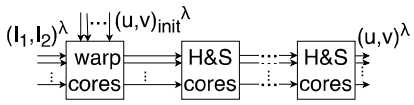

(d) Fully Pipeline Parallel $F_{\pi}$ Mode
Fig. 2. Different Implementations Used

\section{Sum, Up-scaling core}

The sum core is used to sum the velocities calculated in the previous levels with those of the current level. These values are stored in an on chip memory to reduce the interaction with the external memory. Another solution would be to store the velocities in an external memory, but in this case both read and write operations are needed in not neighboured addresses:

- read operations to provide the warp core with the adequate velocities,

- and write operation to merge the new calculated velocities with the ones from the previous level.

This might make the full pipeline of one pyramid level more difficult. Then, results from the sum are extended (up-scaling) in order to be used in the next pyramid level. For up-scaling two FIFO line memory are needed as was made in [8], [9].

\section{Pyramid Creation}

Each image used for each level of the pyramid is being built after the convolution from the coarser pyramid level with a $5 \times 5$ Gaussian kernel (down-sampling) and then stored in the external memory in order to be used for the next steps of the algorithm. Seven FIFOs are used for the pyramid built:

- five for the Gaussian kernel,

- and two to ensure continuous streaming because each coarser level image is four times smaller than the finer level image so for every 4 pixels read from the memory one is written.

\section{E. Pipeline and Parallelism in each Pyramid Level}

It is obvious from the sections above that the up-scaling, warping, $H \& S$ and sum can be performed in a pipeline way for each pyramid level. This has a major advantage: interpolated pixels do not need to be written back to the external memory and then read again, but they can be directly processed by the $H \& S$ core in a pipeline way. It is also possible to compute the optical flow vectors in a fully pipeline parallel way.

\section{DESIGN SPACE EXPLORATION}

As depicted in the previous section, there are a lot of possibilities with the architecture to manage performance. In this sense, a design space exploration has been performed to find the architecture that matches as closely as possible 
the designer's requirements. The exploration is based on the number of the $H \& S$ cores used in our design. Depending on the number of iterations in each level of the pyramid these cores can be used in different modes: iterative $(I)$, partial pipeline $(P)$, fully pipeline $(F)$ and fully pipeline parallel $\left(F_{\pi}\right)$ as shown in Fig 2. These modes have different impacts on computation time. The same cores used in iterative mode in one pyramid level can be used in another mode in a different pyramid level by changing the pipeline depth of the FIFOs memories used and by adding more of them in series or in parallel. For example with respectively 20, 10, 5 iterations for level $_{2}$, level $_{1}$, level $_{0}$ and 10 available cores, then:

- in level $_{2}$, the cores are used in partial pipeline mode,

- in level $_{1}$, in fully pipeline mode with the FIFOs used in level $_{2}$ doubled in depth,

- and in level $_{0}$, in fully pipelined parallel and the number of FIFOs used in level $_{1}$ doubled and used in parallel.

Another critical part is that if in one level $\pi H \& S$ cores are used in parallel then $\pi$ interpolation cores have to be used in parallel for a continuous computation of $\pi$ pixels per clock cycle. The up-scaling and sum components can also be adapted as to be used in parallel mode. We should also mention that when we divide the image width by $\pi$, the residue has to be zero in order to ensure that the $\pi$ parallel pixels are in the same line.

By taking all these into account, depending on the number of cores (I) used and the number of iterations, the total time $T$ for the multi-scale algorithm calculation of the image, without the down-sampling (which takes less than $15 \%$ of the total time), can be estimated by (3).

$$
T=\frac{H e i g h t \cdot W e i g h t \cdot\left(i_{0}+\frac{i_{1}}{4}+\frac{i_{2}}{16}\right)}{f \cdot \Pi}+l a t \cdot \frac{1}{f}
$$

where lat is the latency added from every core which is low regarding the total computation time, $i_{k}$ the iterations for level $l_{k}$ and $f$ the running frequency.

Given the number of iterations $i_{0}$ at level $_{0}$, the total number of logic elements $N$ can be estimated by (4) and (5).

$$
\begin{aligned}
N & =\Pi \cdot N_{H S}+\left\lceil\frac{\Pi}{i_{0}}\right\rceil \cdot\left(N_{\text {warp }}\right)+N_{1} \\
N_{1} & =\left\lceil\frac{\Pi}{i_{0}}\right\rceil \cdot N_{\text {sum }}+\left\lceil\frac{\Pi}{i_{0}}\right\rceil \cdot N_{\text {upscaling }}+N_{\text {downsampling }}
\end{aligned}
$$

where $N_{H S}, N_{\text {warp }}, N_{\text {sum }}, N_{\text {upscaling }}$ and $N_{\text {downsampling }}$ are respectively the numbers of logic elements of $H \& S$, interpolation, sum, up-scaling and down-sampling cores.

The total memory $M$ can also be estimated by (6), (7) and (8). $M_{2}$ represents the extra memory used by $P$ mode in level $_{1}$ to avoid storing the intermediate velocity values in the external memory. This happens because the memory used for sum core for this task is not enough.

$$
\begin{aligned}
M & =\Pi \cdot M_{H S}+M_{\text {warp }}+M_{1}+M_{2} \\
M_{1} & =M_{\text {sum }}+\cdot M_{\text {upscaling }}+M_{\text {downsampling }} \\
M_{2} & =\text { Width } \cdot 2 \cdot \frac{\text { Height } \cdot \text { Weight }}{16}
\end{aligned}
$$

where $M_{H S}, M_{\text {warp }}, M_{\text {sum }}, M_{\text {upscaling }}$ and $M_{\text {downsampling }}$ are respectively the memories used to store values of $H \& S$, warp, sum, up-scaling and down-sampling cores. Width represents the word length depending on the format used $\left(F_{16}, F_{32}\right)$.

The maximum bandwidth of the external memory required is defined by level $l_{0}$ and level $_{1}$ of the pyramid. In level $l_{0}$ the input pixels from the images are 8 bit wide whereas the pixels in level $_{1}$ are depending on the format used $\left(F_{16}, F_{32}\right)$. This happens because the finest level of the pyramid does not get down-sampled. As a result if the calculation in level $_{0}$ is done in $F_{\pi}$ mode with $F_{16}$ then the maximum bandwidth is determined by level $_{0}$, otherwise by level $_{1}$.

TABLE I

MONO-SCALE \& MONO-CORE RESULTS FOR $F_{32}$ AND $F_{16}$

\begin{tabular}{|c|c|c|c|}
\hline core & logic blocks & registers & memory \\
\hline bi-linear interp. $F_{16}$ & $4,543(2 \%)$ & 13,261 & $292,244(<1 \%)$ \\
\hline bi-cubic interp. $F_{16}$ & $17,919(8 \%)$ & 51,375 & $326,876(<1 \%)$ \\
\hline Horn Schunck $F_{16}$ & $4,599(2 \%)$ & 13,759 & $150,352(<1 \%)$ \\
\hline sum $F_{16}$ & $1,841(<1 \%)$ & 5,777 & $9,437,184(18 \%)$ \\
\hline up-scaling $F_{16}$ & $1,768(<1 \%)$ & 2,077 & $67,100(<1 \%)$ \\
\hline down-sampling $F_{16}$ & $13,344(5.6 \%)$ & 23,110 & $94,358(<1 \%)$ \\
\hline \hline bi-linear interp. $F_{32}$ & $7,648(3 \%)$ & 18,261 & $605,644(1 \%)$ \\
\hline bi-cubic interp. $F_{32}$ & $44,991(19 \%)$ & 105,845 & $624,288(1 \%)$ \\
\hline Horn Schunck $F_{32}$ & $9,686(4 \%)$ & 19,720 & $270,483(<1 \%)$ \\
\hline sum $F_{32}$ & $4,351(2 \%)$ & 11,717 & $16,777,216(32 \%)$ \\
\hline up-scaling $F_{32}$ & $2,840(<1 \%)$ & 9,798 & $135,628(1 \%)$ \\
\hline down-sampling $F_{32}$ & $24,387(10 \%)$ & 68,666 & $190,469(<1 \%)$ \\
\hline \multicolumn{4}{|l}{} \\
\hline
\end{tabular}

\section{RESULTS OF IMPLEMENTATION}

For the implementation of the algorithm, the FPGA Altera Stratix V 5SGXEA7H3F35C3 was used. The images from the computer where written to two 64-bit data bus DDR3 memories with a maximum speed of $800 \mathrm{MHz}$ using a PCI express interface. The communication of the external memory with the FPGA was done with the help of two DDR3 SDRAM Controllers with UniPHY provided from Altera. Each DDR3 memory is used for the storage of each image.

In Table I we can see the information about all the key components used in our design. All the components are implemented in VHDL and without DSP to increase clock working frequency. Half and single precision floating point numeric formats are used in the same way as in [19] and all the units are built with the help of the FloPoCo library [20].

In Table II we can see the total resources used regarding the number of $H \& S$ cores, the type of interpolation used and the fps achieved by each implementation. There are also 2 mono-scale fully pipelined versions $\left(\mathrm{v}_{1}\right),\left(\mathrm{v}_{8}\right)$ with half $\left(F_{16}\right)$ and single precision $\left(F_{32}\right)$ floating point format in order to do a fair comparison in the same arithmetic system.

From Table II we can see that by increasing the number of $H \& S$ cores a better fps is achieved. What is also interesting is when for the finest pyramid level 4 pixels per clock cycle are computed then 4 interpolation cores are used as described in section III. That highly impacts the resource usage. For a smaller design the bi-linear interpolation should be chosen for the hardware area to remain small compared to bi-cubic. 
TABLE II

DESIGN SPACE EXPLORATION RESULTS

\begin{tabular}{|c|c|c|c|c|c|c|c|}
\hline Implementation $F_{16}$ & mono-scale $\mathrm{v}_{1}$ & multi-scale $\mathrm{v}_{2}$ & multi-scale $v_{3}$ & multi-scale $\mathrm{v}_{4}$ & multi-scale $\mathrm{v}_{5}$ & multi-scale $v_{6}$ & multi-scale $v_{7}$ \\
\hline Iterations & 20 & $20,10,5$ & $20,10,5$ & $20,10,5$ & $20,10,5$ & $20,10,5$ & $20,10,5$ \\
\hline HS cores $(\Pi)$ & 20 & 1 & 5 & 10 & 20 & 10 & 20 \\
\hline Mode & $F$ & $I^{20}, I^{10}, I^{5}$ & $P^{4}, P^{2}, F$ & $P^{2}, F, F_{2}$ & $F, F_{2}, F_{4}$ & $P, F, F_{2}$ & $F, F_{2}, F_{4}$ \\
\hline interpolation & - & bi-cubic & bi-cubic & bi-cubic & bi-cubic & bi-linear & bi-linear \\
\hline Logic blocks & $93,570(40 \%)$ & $53,531(23 \%)$ & $67,055(29 \%)$ & $109,258(47 \%)$ & $192,386(82 \%)$ & $82,430(35 \%)$ & $148,537(63 \%)$ \\
\hline registers & 250,418 & 150,706 & 189,719 & 293,683 & 529,456 & 208,720 & 382,203 \\
\hline memory & $3,195,788(6 \%)$ & $10,232,738(20 \%)$ & $13,264,090(25 \%)$ & $11,650,850(22 \%)$ & $13,589,222(26 \%)$ & $11,617,698(22 \%)$ & $13,350,693(25 \%)$ \\
\hline frequency & 339 & 276 & 293 & 281 & 270 & 286 & 274 \\
\hline fps & 310 & 29.4 & 149 & 275 & 477 & 277 & 484 \\
\hline Implementation $F_{32}$ & mono-scale $\mathrm{v}_{8}$ & multi-scale $v_{9}$ & multi-scale $\mathrm{v}_{10}$ & multi-scale $v_{11}$ & multi-scale $v_{12}$ & multi-scale $v_{13}$ & multi-scale $\mathrm{v}_{14}$ \\
\hline Iterations & 20 & $20,10,5$ & $20,10,5$ & $20,10,5$ & $20,10,5$ & $20,10,5$ & $20,10,5$ \\
\hline HS cores $(\Pi)$ & 20 & 1 & 5 & 10 & 20 & 10 & 20 \\
\hline Mode & $F$ & $I^{20}, I^{10}, I^{5}$ & $P^{4}, P^{2}, F$ & $P^{2}, F, F_{2}$ & $F, F_{2}, F_{4}$ & $P, F, F_{2}$ & $F, F_{2}, F_{4}$ \\
\hline interpolation & - & bi-cubic & bi-cubic & bi-cubic & bi-cubic & bi-linear & bi-linear \\
\hline Logic blocks & $187,211(80 \%)$ & $98,384(42 \%)$ & $138,012(59 \%)$ & not implementable & not implementable & $168,127(71 \%)$ & not implementable \\
\hline registers & 368413 & 215,633 & 295,138 & - & - & 340,993 & - \\
\hline memory & $5,932,160(11 \%)$ & $17,230,659(33 \%)$ & $23,571,910(45 \%)$ & - & - & $20,854,914(40 \%)$ & - \\
\hline frequency & 209 & 228 & 224 & - & - & 208 & - \\
\hline fps & 191 & 24.3 & 114 & - & - & 202 & - \\
\hline
\end{tabular}

TABLE III

COMPARISON TO STATE-OF-THE-ART IMPLEMENTATION ON FPGA (SORTED ON THE THROUGHPUT)

\begin{tabular}{|c|c|c|c|c|c|c|c|}
\hline Implementation & Algorithm & size & format & $\Pi$ & frame rate & Throughput (Mpixel/s) & Architecture \\
\hline This work $\mathrm{v}_{2}$ & Multi-scale H\&S & $1024 \times 1024$ & $F_{16}$ & 1 & $29.4(4.2)$ & $30.8(4.4)$ & Stratix V 291Mhz (40Mhz) \\
\hline This work $\mathrm{v}_{4}$ & Multi-scale H\&S & $1024 \times 1024$ & $F_{16}$ & 10 & $275(38.8)$ & $288(40.6)$ & Stratix V 296Mhz (40Mhz) \\
\hline This work $\mathrm{v}_{7}$ & Multi-scale H\&S & $1024 \times 1024$ & $F_{16}$ & 20 & $484(70.6)$ & $507(70.4)$ & Stratix V 278Mhz (40Mhz) \\
\hline$(I)[13]$ & Mono-scale $\mathrm{H} \& \mathrm{~S}$ & $640 \times 512$ & $\mathrm{Q}$ & 1 & 30.0 & 9.2 & Stratix IV $295 \mathrm{Mhz}$ \\
\hline$[9]$ & Multi-scale Phase-based & $640 \times 480$ & $Q_{8.0 \rightarrow 8.4}$ & - & 31.5 & 9.6 & Virtex-4 45MHz \\
\hline [8] & Multi-scale L\&K & $640 \times 480$ & $Q_{9.0 \rightarrow 29.8}$ & - & 32.0 & 9.8 & Virtex-4 $83 \mathrm{MHz}$ \\
\hline [16] & Phase-based & $512 \times 512$ & $Q_{8 \rightarrow 12}$ & - & 40.0 & 10.4 & Virtex-4 42MHz \\
\hline [17] & HBM + Refinement & $640 \times 480$ & - & - & 39.0 & 12.0 & Virtex-7 200MHz \\
\hline [10] & Mono-scale L\&K & $800 \times 600$ & $Q_{4.6 \rightarrow 26}$ & - & 170.0 & 81.6 & Virtex-6 $94 \mathrm{MHz}$ \\
\hline$(F)[12]$ & Mono-scale H\&S & $1920 \times 1080$ & $Q_{7.10}$ & 128 & 84.0 & 174.2 & Virtex-7 $150 \mathrm{MHz}$ \\
\hline$(F)[12]$ & Mono-scale H\&S & $1920 \times 1080$ & $F_{32}$ & 32 & 96.5 & 200 & Virtex-7 200MHz \\
\hline$(F)[13]$ & Mono-scale H\&S & $4096 \times 2304$ & Q & 20 & 30.0 & 283.1 & Stratix IV - \\
\hline$[18]$ & Mono-scale L\&K & $1024 \times 1024$ & $Q_{10 \rightarrow 32}$ & - & 1000.0 & 1048 & Virtex-2 $90 \mathrm{MHz}$ \\
\hline
\end{tabular}

If accuracy is the point then bi-cubic interpolation is better. Moreover, we can see that the $\mathrm{v}_{6}$ and $\mathrm{v}_{13}$ have less logic block usage than mono-scale $\mathrm{v}_{1}$ and $\mathrm{v}_{8}$ with small impact in fps. So these 2 designs can replace the mono-scale implementations if memory usage is not important and better range for velocities is needed. Finally, the $F_{32}$ requires more than the double of the resources than the $F_{16}$ meaning that designs $\mathrm{v}_{11}, \mathrm{v}_{12}$ and $\mathrm{v}_{14}$ are not implementable.

In Table III we make a comparison of our works with the state of the art Optical flow algorithms implemented in FPGA. In our works, we have 2 clock frequencies of which the second one is almost the same with the ones used by the previous works in order to do a fair comparison. We can see from this table that $v_{7}$ outperforms in terms of throughput all the other designs except those of Ishii [18]. The reason is that it implements a Mono-scale $L \& K$ algorithm with pseudo-variable frame rate by using two FPGAs, one for the transformation of the serial input to parallel, the other for the calculation of the product sums and a PC for the rest of the steps of the algorithm. Especially now for the $H \& S$ algorithm, we can see that our iterative $\mathrm{v}_{2}, \mathrm{v}_{4}$ and $\mathrm{v}_{7}$ implementations compared to the ones of [13] $I, F$ achieve a $\times 3.34, \times 1.02$ and $\times 1.79$ throughput. Finally we notice that $\mathrm{v}_{4}$ and $\mathrm{v}_{7}$ compared to the other multi-scale implementations [9], [8], [17] achieve a $\times 4.2$, $\times 4.1, \times 3.9$ and $\times 7.3, \times 7.18, \times 6.76$ throughput respectively, even with lower frequency.

\section{CONCLUSION}

We proposed a parametric hierarchical implementation of the gradient Based $H \& S$ motion estimation algorithm in the Stratix V FPGA which, as far as we know, has never been done before. Our exploration showed that we have to switch from single precision to half precision floating point format to fit all the designs we propose. Furthermore we showed that our smallest design can reach $30.8 \mathrm{Mpixel} / \mathrm{s}$ with $23 \%$ usage of the Logic Blocks of the Stratix V and that our fastest outperforms in terms of throughput all the existing state of the art optical flow designs that use solely FPGAs, achieving 507 Mpixel/s with a cost of $63 \%$ of the Stratix V FPGA Logic Blocks. Finally we showed that contrary to the limited range of all the previous $H \& S$ mono-scale designs, all our multi-scale designs achieve a range of 7 for the calculated velocities.

In the future we plan to do more design space exploration on the multi-scale $H \& S$ design by exploring fixed point format, ways to reduce the on-chip memory usage and we also plan to deal with its accuracy. 


\section{REFERENCES}

[1] D.Scharstein and R. Szeliski, "A Taxonomy and Evaluation of Dense Two-Frame Stereo Correspondence Algorithms," International Journal of Computer Vision, vol. 47, pp. 7-42, 2002.

[2] T. Kroeger, R. Timofte, D. Dai, and L. Van Gool, "Fast Optical Flow using Dense Inverse Search," in Computer Vision - ECCV 2016.

[3] A. Petreto, A. Hennequin, T. Koehler, T. Romera, Y. Fargeix, B. Gaillard, M. Bouyer, Q. L. Meunier, and L. Lacassagne, "Energy and Execution Time Comparison of Optical Flow Algorithms on SIMD and GPU architectures," in 2018 Conference on Design and Architectures for Signal and Image Processing (DASIP), 2018, pp. 25-30.

[4] A. Garcia-Dopico, J. Pedraza, M. Nieto, A. Perez, S. Rodrigeuz, and J. Navas, "Parallelization of the optical flow computation in sequences from moving cameras," EURASIP Journal on Image and Video Processing, p. 18, 2014.

[5] A. Plyer, G. Le Besnerais, and F. Champagnat, "Massively parallel Lucas Kanade optical flow for real-time video processing applications," Journal of Real-Time Image Processing, vol. 11, pp. 713-730, 2016.

[6] J. D. Adarve and R. Mahony, "A Filter Formulation for Computing Real Time Optical Flow," IEEE Robotics and Automation Letters, vol. 1, no. 2, pp. 1192-1199, 2016.

[7] T. Senst, R. H. Evangelio, I. Keller, and T. Sikora, "Clustering Motion for Real-Time Optical Flow Based Tracking," in 2012 IEEE Ninth International Conference on Advanced Video and Signal-Based Surveillance, 2012, pp. 410-415.

[8] F. Barranco, M. Tomasi, J. Diaz, M. Vanegas, and E. Ros, "Parallel Architecture for Hierarchical Optical Flow Estimation Based on FPGA," IEEE Transactions on Very Large Scale Integration (VLSI) Systems, vol. 20 , no. 6 , pp. 1058-1067, 2012.

[9] M. Tomasi, M. Vanegas, F. Barranco, J. Diaz, and E. Ros, "HighPerformance Optical-Flow Architecture Based on a Multi-Scale, Multi Orientation Phase-Based Model," IEEE Transactions on Circuits and Systems for Video Technology, vol. 20, no. 12, pp. 1797-1807, 2010

[10] H. Seong, C. E. Rhee, and H. Lee, "A Novel Hardware Architecture of the Lucas-Kanade Optical Flow for Reduced Frame Memory Access," IEEE Transactions on Circuits and Systems for Video Technology, vol. 26, no. 6, pp. 1187-1199, 2016.

[11] R. Rustam, N. H. Hamid, and F. A. Hussin, "FPGA-based hardware implementation of optical flow constraint equation of Horn and Schunck,' in 20124 th International Conference on Intelligent and Advanced Systems (ICIAS 2012), vol. 2, 2012, pp. 790-794.

[12] M. Komorkiewicz, T. Kryjak, and M. Gorgon, "Efficient hardware implementation of the Horn-Schunck algorithm for high-resolution realtime dense optical flow sensor," Sensors, vol. 14, no. 2, pp. 2860-2891, 2014.

[13] M. Kunz, A. Ostrowski, and P. Zipf, "An FPGA-optimized architecture of horn and schunck optical flow algorithm for real-time applications,' in 2014 24th International Conference on Field Programmable Logic and Applications (FPL), 2014, pp. 1-4.

[14] B. K. Horn and B. G. Schunck, "Determining optical flow," Artificial Intelligence, vol. 17 , no. 1 , pp. $185-203,1981$.

[15] M. Tomasi, M. Vanegas, F. Barranco, J. Diaz, and E. Ros, "Real-Time Architecture for a Robust Multi-Scale Stereo Engine on FPGA," IEEE Transactions on Very Large Scale Integration (VLSI) Systems, vol. 20, no. 12, pp. 2208-2219, 2012.

[16] M. Tomasi, M. Vanegas, F. Barranco, J. Daz, and E. Ros, "Massive Parallel-Hardware Architecture for Multiscale Stereo, Optical Flow and Image-Structure Computation," IEEE Transactions on Circuits and Systems for Video Technology, vol. 22, no. 2, pp. 282-294, 2012

[17] K. Seyid, A. Richaud, R. Capoccia, and Y. Leblebici, "FPGA-Based Hardware Implementation of Real-Time Optical Flow Calculation," IEEE Transactions on Circuits and Systems for Video Technology, vol. 28, no. 1, pp. 206-216, 2018.

[18] I. Ishii, T. Taniguchi, K. Yamamoto, and T. Takaki, "High-Frame-Rate Optical Flow System," IEEE Transactions on Circuits and Systems for Video Technology, vol. 22, no. 1, pp. 105-112, 2012.

[19] S. Piskorski, L. Lacassagne, S. Bouaziz, and D. Etiemble, "Customizing CPU Instructions for Embedded Vision Systems," in 2006 International Workshop on Computer Architecture for Machine Perception and Sensing, 2006, pp. 59-64.

[20] F. de Dinechin and B. Pasca, "Designing Custom Arithmetic Data Paths with FloPoCo," IEEE Design Test of Computers, vol. 28, no. 4, pp. 18-27, 2011. 